\title{
Boson stars with nonminimal coupling
}

\author{
Anja Marunović \\ Institute for Theoretical Physics, Spinoza Institute and Center for Extreme Matter and \\ Emergent Phenomena, Utrecht University, Postbus 80.195, 3508 TD Utrecht, The Netherlands \\ Email: a.marunovic@uu.nl
}

\begin{abstract}
Boson stars coupled to Einsteins general relativity possess some features similar to gravastars, such as the anisotropy in principal pressures and relatively large compactness $\left(\mu_{\max }=0.32\right)$. However, no matter how large the self-interaction is, the ordinary boson star cannot obtain arbitrarily large compression and as such does not represent a good black hole mimicker. When the boson star is nonminimally coupled to gravity, the resulting configurations resemble more the dark energy stars then the ordinary boson stars, with compactness significantly larger then that in ordinary boson stars (if matter is not constrained with the energy conditions). The gravitationally bound system of a boson star and a global monopole represents a good black hole mimicker.
\end{abstract}

\section{Introduction}

If matter obeys the strong energy condition (SEC), classical general relativity predicts the existence of black holes. (According to the SEC the sum of the energy density and pressures, $\rho+p_{i} \geq 0$, cannot be negative.) Black holes are stationary, vacuum solutions of the Einstein equations that possess an event horizon and a physical singularity which is hidden by the event horizon. This physical singularity represents an as-yet-unresolved problem, since it implies the controversial information loss paradox according to which any information will get completely lost on the singularity of a black hole. The principles of information loss are in conflict with the standard laws of quantum physics. Taking all these considerations into account it is natural to question whether the final stage of a massive star collapse is a black hole, or perhaps some other as-yet-not-understood dense object, that prevents further collapse.

Sakharov was the first that introduced the concept of nonsingular collapse through the equation of state for the cosmological dark energy (for which the pressure is negative, $p=-\rho$ ) as a super-dense fluid and then Gliner assumed that such a fluid could be the final state of the gravitational collapse. Inspired by these ideas Mazur and Mottola investigated alternative configurations which led to a solution dubbed the gravastar (gravitational vacuum star) $\stackrel{1}{*}$. This anisotropic, highly compact astrophysical object consists of a de Sitter core, which through a vacuum transition layer matches on an exterior Schwarzschild space-time by avoiding an event horizon formation. Due to their high compactness (defined as the ratio of the mass to radius) gravastars are perceived by distant observers as black holes, and hence they can be good black hole mimickers.

Apart from the de Sitter core, gravastars possess the peculiar property of pressure anisotropy ${ }_{2}^{2}$. Microscopic models of gravastars would shed light on the above mentioned problems of curvature singularities and black hole information loss para- 
dox.

\section{Boson stars nonminimally coupled to gravity}

The oldest, and accordingly the most studied, astrophysical example based on the Lagrangian formalism, is the boson star, which is a compact object built from a self-interacting, gravitationally bound scalar field. It is known that boson stars coupled to Einsteins general relativity possess some features that characterize gravastars, such as the anisotropy in principal pressures and relatively large compactness $\left(\mu_{\max }=0.32\right)$. Here we extend the analysis of boson stars and modify the EinsteinHilbert action by introducing a nonminimal coupling of the scalar field to gravity via the Ricci curvature scalar $\underline{3}$ :

$$
S_{\phi}=\int d^{4} x \sqrt{-g}\left(-g^{\mu \nu} \partial_{\mu} \phi^{*} \partial_{\nu} \phi-m^{2} \phi^{*} \phi-\frac{\lambda_{\phi}}{2}\left(\phi^{*} \phi\right)^{2}+\xi R \phi^{*} \phi\right),
$$
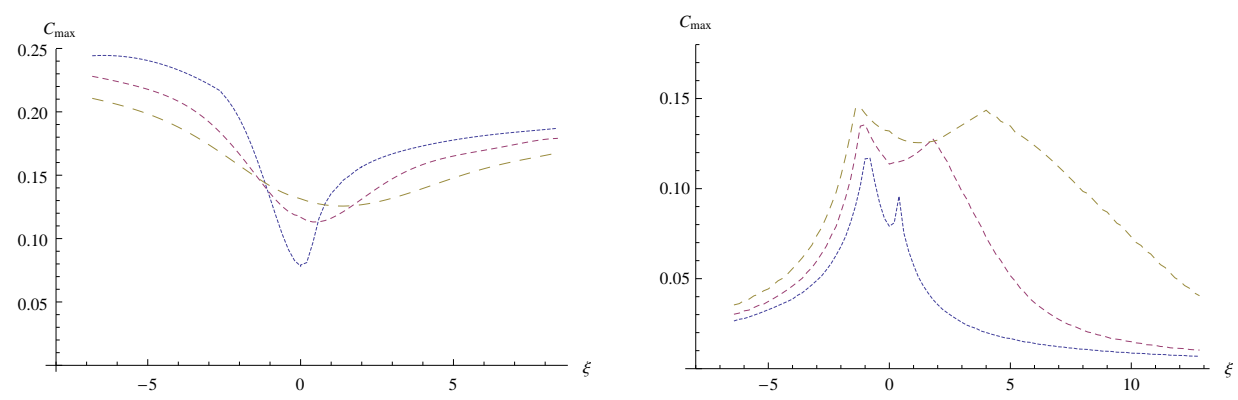

Fig. 1. Effective compactness as a function of $\xi$ for $\lambda_{\phi}=0$ (dotted curve), $\lambda_{\phi}=20$ (short-dashed) and $\lambda_{\phi}=50$ (long-dashed). Right: Effective compactness if weak energy condition (WEC) and dominant energy condition (DEC) are obeyed.

We show that already this minimal extension of general relativity results in configurations that resemble more the dark energy stars (due to negative pressures) then the ordinary boson stars (see Fig. 2), with compactness 6 significantly larger then that in ordinary boson stars (if matter is not constrained with the energy conditions) (see Fig (1). It is interesting to observe that, if matter is not constrained with the energy conditions, $\mu_{\max } \approx 0.5$ in the region $\xi<0$ where the pressures are negative. However, if matter obeys the energy conditions, $\mu_{\max } \approx 0.32$, and hence not much larger then in the case of ordinary boson stars.

a The metric is spherically symmetric $d s^{2}=-e^{\nu(r)} d t^{2}+e^{\lambda(r)} d r^{2}+r^{2} d \theta^{2}+r^{2} \sin ^{2} \theta d \varphi^{2}$.

${ }^{\mathrm{b}}$ Effective compactness is defined as $C_{\text {eff }}=M_{99} / R_{99}$ where $R_{99}$ is the radius at which the mass, defined in terms of the metric function $e^{-\lambda}=1-2 m / r$ equals $99 \%$ of the total mass $M=m(\infty)$. Compactness is $\mu=2 m / r=2 C_{e f f}$. 

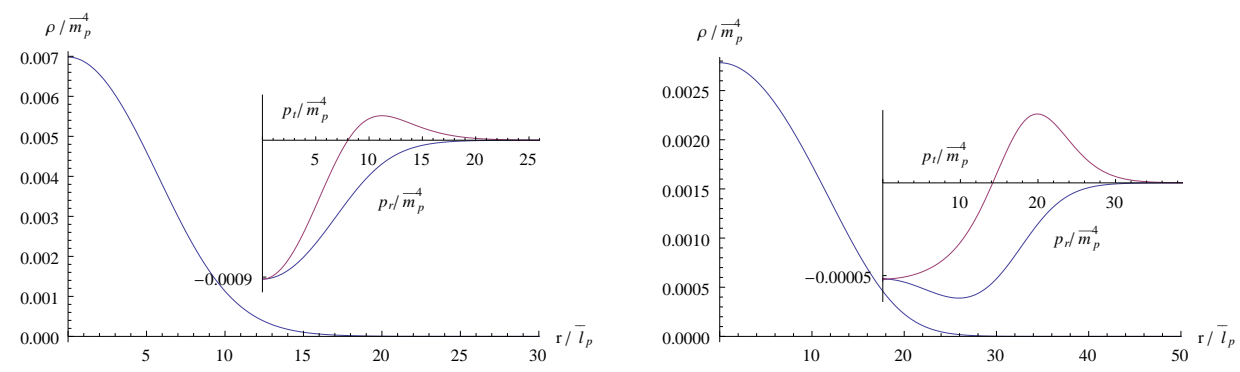

Fig. 2. Energy density and pressures (inset) for: a) Left: $\xi=-4,\left\{\lambda_{\phi}, \sigma_{c}\right\}=\{0,0.050\}$; b) Right: $\xi=-4,\left\{\lambda_{\phi}, \sigma_{c}\right\}=\{100,0.034\}$.

\section{Boson defect stars}

Another field-theoretic model that we investigate involves a combined system of a boson star and a global monopole ${ }^{4}$. The simplest field-theoretic realization of the global monopole includes a scalar field theory with an (global) $O(3)$ - symmetry which is spontaneously broken to $O(2)$ by the vacuum.

The action that governs the dynamics of a global monopole is:

$$
S_{\phi}=\int d^{4} x \sqrt{-g}\left(-\frac{1}{2} g^{\mu \nu} \partial_{\mu}\left(\phi^{a}\right) \partial_{\nu}\left(\phi^{a}\right)-V\left(\phi^{a}\right)+\frac{1}{2} \xi R\left(\phi^{a} \phi^{a}\right)\right)
$$

and the potential is a standard symmetry breaking Mexican hat potential,

$$
V\left(\phi^{a} \phi^{a}\right)=\frac{\mu^{2}}{2} \phi^{a} \phi^{a}+\frac{\lambda}{4}\left(\phi^{a} \phi^{a}\right)^{2}+\frac{\mu^{4}}{4 \lambda} .
$$

A global monopole is a solution of the field for the given action, with the so called hedgehog Ansatz

$$
\phi^{a}=\phi(r)(\sin \theta \cos \varphi, \sin \theta \sin \varphi, \cos \varphi) .
$$

It is a well known result that global monopoles in a very simplified model in which the field profile is modeled with a $\Theta$-function, exhibit a de Sitter interior $\left(p_{r, t}(0)=-\rho(0)\right)$ and a Schwarzschild exterior with a deficit solid angle $1-\Delta-$ $2 G M / r$. The deficit solid angle is $\Delta=8 \pi G_{N} \phi_{0}^{2}$ and denotes the scale of the symmetry breaking; $\phi_{0}$ is the vacuum expectation value of the field (minimum of the potential). Due to the de Sitter core, global monopoles are gravitationally repulsive. Introducing nonminimal coupling, the deficit solid angle is modified $\tilde{\Delta}=\Delta /(1+\xi \Delta)$, effective force could be locally attractive and bound circular orbits around monopole could exist.

We show that a repulsive monopole stabilizes an attractive boson star and the resulting configuration exhibits large energy density, large (and negative) principal pressures, large compactness, large effective potential, large local forces, and yet exhibit no event horizon (see Fig. 3). As such a composite system of a boson star and a global monopole represents a convincing microscopic candidate for a black hole mimicker. 

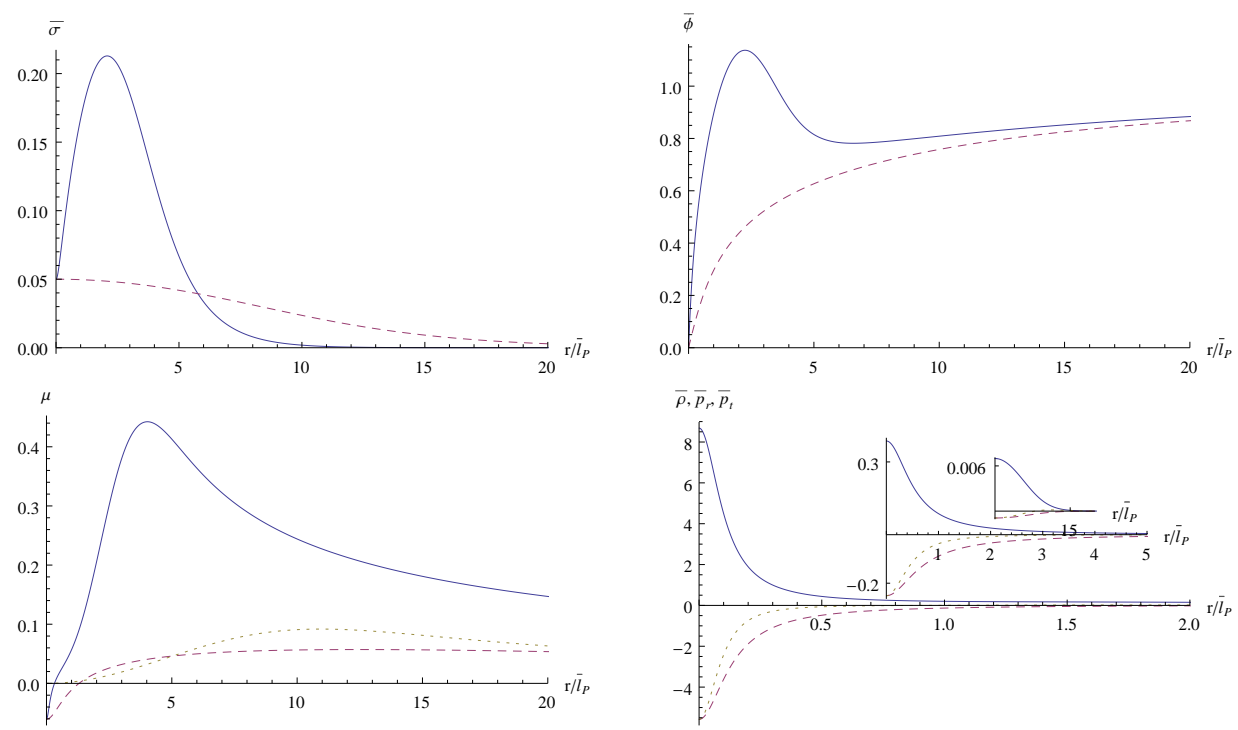

Fig. 3. Upper left: The boson star field alone (dashed) and in the presence of a global monopole (solid). Upper right: The global monopole field alone (dashed) and in the presence of a boson star (solid). Lower left: Compactness for the boson star alone (dotted), a global monopole alone (dashed) and a combined system (solid). Lower right: Energy density (solid) of the / combined system / global monopole (inset) / boson star (smaller inset); radial pressure (dashed) and transversal pressure (dotted). $\sigma_{0}=0.05, \lambda_{\mathrm{BS}}=0, \xi_{\mathrm{BS}}=-4, \lambda_{\mathrm{GM}}=0.1, \Delta=0.08, \xi_{\mathrm{GM}}=5$.

\section{References}

1. P. O. Mazur and E. Mottola, "Gravitational Condensate Stars: An Alternative to Black Holes", Proc. Nat. Acad. Sci. 101 (2004) 9545, arxiv:gr-qc/0109035; "Surface Tension and Negative Pressure Interior of a Non-Singular 'Black Hole"', [arxiv: 1501.03806].

2. A. DeBenedictis, D. Horvat, S. Ilijić, S. Kloster, K. S. Viswanathan, "Gravastar Solutions with Continuous Pressures and Equation of State", Class. Quant. Grav. 23 (2006) 2303-2316, arxiv:gr-qc/0512054.

3. Dubravko Horvat and Anja Marunović, "Dark energy-like stars from nonminimally coupled scalar field", Class. Quantum Grav. 30145006 2013, arXiv:1212.3781.

4. Anja Marunović and Miljenko Murković, "A novel black hole mimicker: a boson star and a global monopole nonminimally coupled to gravity", Class. Quantum Grav. 31 (2014) 045010, arXiv:1308.6489. 